\title{
Communication and Advanced Metering Issues for the Incorporation of Renewable Energy Resources in Microgrids
}

\author{
Alexander Blandón ${ }^{1}$, Nicolás Muñoz-Galeano², Juan B. Cano-Quintero³, \\ Jesús M. López-Lezama ${ }^{4}$, Fernando Villada-Duque ${ }^{5}$ \\ ${ }^{1}$ Department of Electrical Engineering, Research Group GIMEl, University of Antioquia (UdeA), Calle 67 No 53-108, Medellin, \\ Colombia \\ ${ }^{2}$ Department of Electrical Engineering, Research Group GIMEl, University of Antioquia (UdeA), Calle 67 No 53-108, Medellín, \\ Colombia \\ ${ }^{3}$ Department of Electrical Engineering, Research Group GIMEl, University of Antioquia (UdeA), Calle 67 No 53-108, Medellín, \\ Colombia \\ ${ }^{4}$ Department of Electrical Engineering, Research Group GIMEl, University of Antioquia (UdeA), Calle 67 No 53-108, Medellin, \\ Colombia \\ ${ }^{5}$ Department of Electrical Engineering, Research Group GIMEl, University of Antioquia (UdeA), Calle 67 No 53-108, Medellin, \\ Colombia
}

\begin{abstract}
The ever-growing demand for electricity and a greater awareness of environmental issues have boosted the use of renewable energy resources within distribution networks. In this context, microgrids have allowed the integration of this type of generation along with an active role of users. Nevertheless, pursuing a massive integration of renewable energy resources under the concept of microgrids requires addressing several issues in advanced metering and communication. This paper presents a comprehensive review which includes both wired and wireless communications. Wired communication is focused on Power Line Communication (PLC), while wireless communication is focused on Low-Power Wide-Area Network (LPWAN), emphasizing on Sigfox, LoRa and NB-IoT. Also, the most relevant issues for the implementation of advanced metering in microgrids are presented and explained.
\end{abstract}

Keywords: Microgrids, Advanced Metering, Communication, PLC, LPWAN.

\section{INTRODUCTION}

In traditional power systems electricity is generated in centralized power plants and transmitted over high-voltage transmission lines to be delivered to end-users and distribution systems. In this classic model the power flows in one direction, from generation to transmission lines and from these to distribution networks and ultimately to end-users. Nevertheless, in the last decade the electric industry has experienced a change towards bidirectional power flows due to the ever-growing integration of small-scale generation in distribution networks [1], [2]. This has brought new challenges in multiple aspects since regulatory frameworks, business models, operating standards, as well as planning and investment approaches have all been design bearing in mind the traditional paradigm [3], [4].

Technological advances in small-scale generation technologies, as well as the desire to reduce dependence on coal-based generation, have been the main drivers for the growing participation of renewable energy generation in the power grid [5]. In this context, microgrids have played a key role, since they allow the integration of distributed energy resources (DERs) which include distributed generation (DG), demand response (DR) and storage systems [6], [7].

Microgrids propitiate the integration of DERs, making power systems more efficient and providing a better management of energy resources [8], [9]. As regards its operation mode, a microgrid can operate connected to main grid or in islanded mode. In the connected mode, the owners of DG units may sell their energy surplus to the main grid. In islanded mode, the main objective is to achieve energy balance between DERs and microgrid loads. A sophisticated communication system is required for both operation modes and also for guaranteeing reliability, security and power quality. DERs and microgrid loads required a constant monitoring of electrical variables. This information is provided to a control system which appropriately regulates bidirectional power flows.

Communication in microgrids can be faced by using two kinds of technologies: wired and wireless communications. In regards to wired communication, the most widespread technology is Power Line Communication (PLC) which uses the existing power lines for transmitting information. Concerning wireless technology, there is a great variety of possibilities [10],[11]. This paper considers the emerging Low-Power Wide-Area Network (LPWAN) technology, which allows long-range communications at a low bit rate among microgrid smart meters [12],[13]. LPWAN is mainly used for Internet of Things (IoT); however, it can be easily adapted for microgrid applications. As stated in several studies LPWAN features certain advantages for its 
implementation in microgrids that include low power consumption, long operation range, and low cost [14-19].

The LPWAN technologies considered in this paper are: Sigfox, LoRa and NB-IoT. A comparison among these technologies is presented in terms of several issues such as battery duration, latency, quality of service, implementation complexity and system cost, among others. Also, the most relevant issues for the implementation of advanced metering in microgrids are discussed.

This paper is organized as follows: Section II covers the aspects related to communication in microgrids, including wired and wireless communications. Section III presents a comparison among LPWAN technologies. Section IV highlights important aspects when advanced metering is incorporated in microgrids. Section IV concludes and discusses the most relevant aspects.

\section{COMMUNICATION IN MICROGRIDS}

The operational control and protection of a microgrid requires a sophisticated communication infrastructure able to operate DG, facilitate DR and coordinate the system protection. In terms of control, communication and protection microgrids can be designed in a centralized or decentralized architecture [20].

\section{A. $\quad$ Wired Communications}

PLC technology uses the power distribution lines as a wired communication channel. Power lines are not designed for communications, resulting in noise and distortion issues that limits speed and performance. However PLC requires less new infrastructure to be implemented effectively lowering implementation costs [21].

Different PLC applications on microgrids or smart metering have been reported in the specialized literature. In Italy, starting with the "Telegestore" project [22], smart metering has been implemented using narrow band PLC (NB-PLC). The "linky project" [23] implemented over 25.000 smart energy meters in France using PLC communication. This project finished in 2011 and currently a massive installation of smart meters using PLC is scheduled [24]. In other countries such as Spain, Sweden, UK, smart metering applications have been implemented by mixing PLC with other wired or wireless techniques [24].

PLC has been implemented on many home automation applications (i.e the X10 and UPB -Universal Powerline Busprotocols, among others). By this, PLC is commonly used on applications combining home automation and smart metering [25]. The use of smart metering data in home automation applications has been identified as a promising energy saving opportunity [26],[27].

Several applications of PLC on smart grid have been reported. In [28] the authors propose a new implementation of PLC in DC microgrids, where data is shared by using frequency shift modulation (FSM). FSM consists on changing the commutation frequency of the $\mathrm{DC} / \mathrm{DC}$ converters to transmit information. Due to output ripple, this frequency change can be measured by the rest of microgrid devices connected to the same DC bus. This approach allows system simplification, not needing separated frequencies signals for power switching and communications. In [29] PLC is used as communication method for energy management in a DC microgrid. In [30] a monitoring system for an AC microgrid was implemented using NB-PLC. In [31] a DC microgrid using two photovoltaic sources is described realizing load voltage regulation by using PLC communications.

\section{B. $\quad$ Wireless Communications}

Recently, wireless communication has become an essential feature of microgrids for synchronizing the operation among different devices. As an advantage, wireless communication is faster than PLC. Another advantage is that wireless communication is a way of transmitting information from one point to another, without using any connection like wires, cables or any physical medium. All microgrids devices which are distributed over an extensive area can be easily connected through wireless communication.

Data transportation technology is nowadays framed with meaningful technical differences between transmission speed and data quantity that can be transmitted. These two characteristics are inversely proportional when defining which technology can be used in microgrid applications or even for large-scale networks such as smart cities. Then, it is necessary to define the technology that best fits the specific needs of the application and that fulfills the requirements in terms or speed, bandwidth and quality. LPWAN technology was chosen due to its low consumption of power, long range of operation, and low cost [14]. LPWAN can reach between one to five kilometers in urban zones of range which is enough for microgrid applications [15]. Also, the duration of its battery is around ten years, if it is considered a typical microgrid with twenty years of useful life, maintenance engineers only have to make a replacement which is not expensive [16]. LPWAN technology has been proven for numerous engineers and researchers around the world having great performance not only in outdoor but also indoor environments [14-19]. In general terms, LPWAN is more than suitable for communication in microgrids which does not require huge amounts of data transmission or a long range of operation. In this case, three LPWAN technologies for the development of communications for microgrids are analyzed: Sigfox, LoRa and NB-IoT.

Sigfox is a LPWAN technology operating company that develops and trades its own solution. It was created in 2010 in Toulouse, France, and it actually operates in over 31 countries, in partnership with network operators and spreading its base proprietary stations with radios connected to IP servers, the final devices connect to these stations using modulation by binary phase shift keying (BPSK) with ultranarrow band, only $100 \mathrm{~Hz}$, in industrial, scientific and medical (ISM) radio bands, which are bands without licenses in different regions of the world; for example, $915 \mathrm{MHz}$ in America, $868 \mathrm{MHz}$ in Europe, and $433 \mathrm{MHz}$ in Asia. Sigfox 
has a high noise immunity, low energy consumption, high receptor sensitivity and a low cost antenna, since it works in a ultra-narrow band but it otherwise has a maximum performance of $100 \mathrm{bps}$, which represents a disadvantage in terms of the quantity of information that can be transmitted. Initially, Sigfox only allowed sending data upwards, it means from terminal devices to the base station, but this evolved to limited bidirectional communication [32]. Downlinks could only happen after uplinks, and uplinks are limited to 140 messages a day of 12 bytes maximum. On the contrary, downlinks are limited to 4 messages a day of 8 bytes maximum, which means that it is not possible to validate if all upward messages were delivered successfully and if information was delivered fault-free.

Communication trustworthiness is improved using more time, diversifying frequencies and duplicating transmissions. Usually, the total bandwidth is divided into several channels of $100 \mathrm{~Hz}$, each upward message is sent three times through different frequency channels, and each terminal device can send the upward message through different random channels. The messages of the final devices can be received simultaneously through different channels in the base station.

Lora is a modulation technology located in the physical layer. It was initially developed by the company SEMTECH. Nowadays, LoRa Alliance is a non-profit organization formed by different companies committed to the massification of this technology and that have worked together in the development of LoRaWAN (since 2015), which is a network protocol based on the LoRa modules. These modules work in ISM bands with frequencies inferior to $1 \mathrm{GHz}$, and likewise Sigfox; $915 \mathrm{MHz}$ in America, $868 \mathrm{MHz}$ in Europe and 433 $\mathrm{MHz}$ in Asia [33]. The communication is bidirectional with chirp spread spectrum (CSS) modulation that keeps a narrowband signal in a wider-band channel compared to Sigfox, which results in a signal with less noise levels, with high immunity to interferences, and very had to be located by intruders [34], [35].

LoRa uses six spreading factors (SF7 - SF12), with which higher ranges can be achieved but with less data speed, the higher SF the higher range but less data speed and vice versa. Using LoRa, 300bps to 50kbps speed can be achieved, but this depends on the SF and the bandwidth of the channel. Usually, in the LoRaWAN protocol, the message is sent and received by all final devices, but having one receiver's address, only this final device, to which the message was sent, will attend it and will send a confirmation message to the base station (LoRa Gateway), the base station will retransmit this message to the application server using any other type of protocol such as TCP-IP, WIFI, GSM, Bluetooth among others [36], [37].

There are three types of final devices, which depend of the amount of time they should be in communication with the Gateway:

- Type A final device, they are devices with bidirectional communication in which the final device sends a message to the Gateway and then opens a reception window, that has two short periods of time to receive downward messages. This type of devices is designed for those terminals that only require sending information to the base station at certain times, which means in a very low energy consumption and long duration batteries (up to 10 years).

- Type B final devices, they are devices with bidirectional communication which have additional communication programmed windows to the communication windows in type A devices, with this, it is possible that final devices receive group messages from the application server and these can acknowledge receipt, allowing the server to know that the final devices are in listening mode.

- Type C final devices, these types of devices remain in listening mode all the time (as receiver) and only stop listening when they are going to transmit, they are ideal to constant monitoring systems, and they are suitable for not using batteries and being constantly connected to the electrical network given that their consumption is higher.

NB-IoT is a narrow band technology released by 3GPP group in June 2016. It is a technology that uses cell phone networks, for which it takes frequency bands that are licensed in GSM and LTE networks. It has a bandwidth up to $200 \mathrm{KHz}$ and it is based on the LTE protocol, it even reduces the functionality of this protocol to the lowest and it can get to enhance it according to the IoT application need [38].

NB-IotT allows connectivity up to 100.000 final devices per cell and it has the capacity to spread much more using more carriers.

NB-IoT uses frequency-division multiple access (FDMA) modulation for upward messages and orthogonal frequencydivision multiple access (OFDMA) modulation for downward messages. NB-IoT can use up to 200kbps for downlinks and $20 \mathrm{kbps}$ for uplinks with a maximum length of 1600 bytes per message, but this is detrimental to the battery duration for isolated applications. However, NB-IoT batteries can last up to 10 years when up to 200 bytes are transmitted a day [39].

\section{COMPARISON OF LPWAN EMERGING TECHNOLOGIES}

The three above-LPWAN technologies can be compared in terms of some important factors such as: battery duration, latency, messages length, terminal devices quantity, coverage, quality of service, implementation complexity and system cost [14], [40].

In terms of the battery duration, the final devices of the three technologies allow a sleep mode most of the time, with very low energy consumption. But NB-IoT requires a higher energy consumption since its communication with the base is synchronous and has higher quality of service (QoS) and higher bandwidth which reduces the duration of the battery in contrast with LoRa (type A and B) and Sigfox. Nevertheless, LoRa type $\mathrm{C}$ devices require much more energy as it is thought for applications connected to the electrical network and to remain in constant transmission [41].

Latency is a factor that influences the quality of service; the lower the latency, the fastest the response, but low latency requires higher bandwidth in the transmission, higher power consumption, and less spreading distance. In this regard, NB- 
IoT has a great advantage in comparison to LoRa and Sigfox, since using cellphone networks, the LTE protocol and and broader bandwidth, it can have transmissions in less time with a great amount of data.

Messages length (payload length) is a very important feature when defining which technology to use. If the information that will be transmitted is very limited and occasional, the best protocol is Sigfox in which 12-byte upward frames (up to 140 messages a day) and 8-byte downward frames (up to 4 messages a day) can be sent. On the contrary, LoRa allows a maximum of 243 bytes per message limitless a day; in contrast to NB-IoT that allows 1600 bytes with no limitations a day as well.

In regards to terminal devices, the best performance is fulfilled by NB-IoT which allows up to 100.000 devices registered to one cell. In contrast to LoRa and Sigfox which allows 50.000 devices registered to one network. However, the coverage of the base stations should be taken into account. For urban applications, Sigfox has a range up to $10 \mathrm{Kms}$, LoRa $5 \mathrm{Kms}$ and NB-IoT $1 \mathrm{Km}$; but in rural applications with line of sight, Sigfox can get up to $40 \mathrm{Kms}$, LoRa $20 \mathrm{Kms}$ and NB-IoT $10 \mathrm{Kms}$. Noticing that NB-IoT is not designed for suburban or rural areas that do not have LTE cellphone operators coverage.

For the implementation of any of these systems, the level of maturity of such technology should be considered as well as its technical support. For example, Sigfox is being implemented in many countries with the support of the brand. LoRa is developing more massively with the LoRaWAN standard in which private or public LP-WAN networks can be created with the support of companies and free servers for the management of the network and final devices. Likewise Sigfox and LoRa developments are still growing, while NBIoT is much more recent, it still requires higher development but has the support of some cellphone multinational companies that are committed to the massification of this technology for IoT.

One of the key factors when defining the use of an IoT technology is the cost. First, the cost for spectrum use should be taken into account. NB-IoT used frequency bands that are paid, compared to LoRa and Sigfox that use ISM bands that are free frequency bands, but with some limitations. Additionally, the hardware cost must also be considered (base stations and final devices). In this regard, a NB-IoT development is much more expensive than LoRa and Sigfox (see Table 1), but each system has its own benefits that allow the user to choose according to their project characteristics.

Table I: Hardware costs for LoRa, Sigfox and NB-IoT [14].

\begin{tabular}{|l|l|l|l|}
\hline & $\begin{array}{l}\text { Spectrum } \\
\text { cost }\end{array}$ & $\begin{array}{l}\text { Final devices } \\
\text { cost }\end{array}$ & Development cost \\
\hline LoRa & Free & $3-5$ USD & $>100$ USD/ base station \\
\hline Sigfox & Free & $<3$ USD & $>4000 \mathrm{USD} /$ base station \\
\hline NB-IoT & $\begin{array}{l}>500 \mathrm{million} \\
\text { USD } / \mathrm{MHz}\end{array}$ & $>20 \mathrm{USD}$ & $>15000 \mathrm{USD} /$ base station \\
\hline
\end{tabular}

\section{ADVANCED METERING ISSUES}

The objective of this section is to present the most relevant issues for the implementation of advanced metering in microgrids [42-46]. Based on the literature review, the following questions are answered: $A$ ) Why is advanced metering in microgrids needed?, $B$ ) Where is this advanced metering needed?, $C$ ) What are the benefits of advanced metering in microgrids?, D) Can advanced energy meters monitor bidirectional power flows in microgrids?, E) What is the immediate future of microgrids?, $F$ ) What is the importance of Advanced Metering Infrastructure (AMI)?, G) What are the international standards for advanced metering?, $H$ ) What is zero-energy buildings and their relationship with advanced metering?, I) What are the main characteristics of an advanced metering system?, $J$ ) What are the types of data storage for advanced metering systems?, K) What are distributed metering systems?

\section{A. $\quad$ Why is advanced metering in microgrids needed?}

Advanced metering is a fundamental part of a microgrid. This technology not only allows measuring the electricity consumption but also having information about other grid parameters, such as voltage, current and power factor, active power, reactive power, harmonics among others. These parameters are equally important to manage a microgrid since they allow the operator to know the status of the grid, the power fluxes, the possible failures of the system, and the consumption history. They also make it possible to create individual load profiles for each particular client, their consumption tendencies and even to propose the user possible enhancements in their consumption habits for a rational use of electricity [41].

\section{B. $\quad$ Where is this advanced metering needed?}

In smart cities and micro grids, it is absolutely necessary the use of advanced metering, since it is with this application that the network operator can manage almost all the system. Taking advantage of the communication with advanced meters, it is possible to monitor the system constant, even the operator can disconnect and connect loads in every meter (only if the meter has this capacity), which avoids operators moving staff, reducing the required resources for the network/grid maintenance. But, additionally, having the control over segregated loads, the operator can decide to disconnect loads that are not sensitive in moments when generation can be insufficient for the demand or the operator can even use the history load profiles to determine which generators are needed for the projected demand, taking into account every type of generation expenses and the hours of major use of electricity, which may result in less expenses and more energetic efficiency.

\section{What are the benefits of advanced metering in microgrids?}

Using advanced meters in microgrids, along with an analysis 
and management software, brings the following benefits: 1) Timetable profiles with which it is possible to identify the hours of more consumption, as well as maximum consumptions which allow programming the use of equipment and systems to prevent over charges to the electrical systems and reduce maintenance costs. 2) Comparisons of consumptions (benchmarking) that help to implement better energetic consumption practices, comparing current trends with past ones as well as using statistical data of other installations that are in similar conditions; through this information analysis, enhancement actions for an efficient electric energy use can be carried out. 3) Management, allocation and energetic expenses analysis in buildings with micro grids and advanced metering allow programming activities in hours in which energy has a lower cost and it is even possible to validate when it is more efficient to consume the energy generated in a microgrid or if is profitable to deliver such generated energy to the external grid, with the economic profits for the owner of the building. 4) Power quality is another benefit that can be obtained from micro grids with advanced metering, given that these meters can get data that affect the power quality such as sudden power outages, under voltage, overvoltage, over current, harmonics, power factor and reactive energy. All of these factors seriously affect the durability of the installation, as well as its own maintenance.

\section{D. $\quad$ Can advanced energy meters monitor bidirectional} power flows in microgrids?

Yes, advanced energy meters can monitor the bidirectional flows of energy in microgrids. Current electrical grids are interconnected and complex systems in which there are power flows in different directions, since the arrival of microgrids that usually count with DERs systems, that bring along a new dynamic to the grids since they incorporate new sources of energy that network operators should be able to monitor and control so those power flows do not end up saturating or collapsing the grid. This new scenario demands news systems that allow the electrical grid to be actually smart through the management of the bi-directionality and the changing flow of energy, that is why energy advanced meters are required. In addition, those systems must be compatible with both new and old systems.

\section{E. What is the immediate future of microgrids?}

One idea of future microgrids is the use and integration in all nodes of the electric grid, using advanced meters to provide the needed information about local energy consumption, energy flows and energy quality variables that help the network operator to make better decisions, and even step ahead a possible system failure. It is in this regard when IoT (Internet of Things) can provide new opportunities to the development of advanced power meters. In addition, by having nodes with sensors that can interact with the energy meters, it is possible to establish a network of elements capable of acting over different network points. This information can be transmitted to any part of the world via internet. In the future, advanced meters should be able to attend a group of nodes or a micro grid to search and control power flows by using artificial intelligence, by learning and redesigning algorithms of efficient management power flows, and by learning to handle the difficulties that may come out in the grid, so that human intervention is minimally required.

\section{F. What is the importance of Advanced Metering Infrastructure (AMI)?}

AMI architecture provides systems with measurements and actuators that can be controlled by the network operator, this can be done by using energy meters as gateways to other subsystems such as water or gas meters, capable of communicating short distances and low power to send messages to the energy meter. This is capable of communication low-power wide-area network (LPWAN) to link with the gateways or base stations that can retransmit the information via internet or by cellular networks to a central access system unit. This unit can be equipped with web interfaces to monitor and control the network nodes, and the capacity to implement a management strategy of power flows management and send instructions to the network actuators to keep the system stable. Communication could be bidirectional, but it is normally Half-duplex when low-power final elements are at stake. It means that when a final device is transmitting, it could not get messages, and when it is getting messages it could not transmit. This is detrimental for the reply speed of the systems, but it keeps a balance between communication capability and efficient use of energy, especially for battery-based devices.

\section{G. What are the international standards for advanced metering?}

Advanced metering systems should comply with some international standards. For example, ISO/IEC/IEEE 21451 for treatment, sign processing and data structure in transductors; IEC 61000-4 electromagnetic compatibility; IEC 62052-11 applied to measurement equipment tests in AC; IEC 62053-21 particular requisites for static counters of active energy (type 1 and 2); IEEE 1459-2010 definitions for the measurement of electric energy quantity in sinusoidal conditions, non-sinusoidal, balanced or unbalanced; IED 60364-1 which includes recommendations of energetic efficiency in low voltage electrical installations.

\section{H. What is zero-energy buildings and their relationship with advanced metering?}

A zero-energy building can be defined as an almost zero electricity consumption installation; given that it has a generation system with non-conventional DERs or cogeneration and that the total quantity of consumed energy in a year is likely to be the same amount of energy generated in the same year. The use of advanced meters is very important for this type of building in order to manage and control energy flows. Advanced metering systems monitor, supervise, 
International Journal of Engineering Research and Technology. ISSN 0974-3154, Volume 13, Number 9 (2020), pp. 2196-2204

(C) International Research Publication House. https://dx.doi.org/10.37624/IJERT/13.9.2020.2196-2204

visualize and manage the loads by using a central supervision center.

I. What are the main characteristics of an advanced metering system?

An advanced meter should comply with at least the following characteristics: capability of acquiring data, capability of communication, capability of data visualization and data storage. This is why there are different types of advanced meters:

-Energy meter: it meters the quantity of consumed energy or delivered to the grid

-Power meter: it meters voltage, current and powers

-Power analyzer: it manages active and reactive power; it meters voltage, current, power factor and energy

-Quality energy analyzer: it analyzes power and measures frequency, total harmonic distortion (THD), voltage irregularities and disturbances. The THD distributed evaluation allows the identification of disturbing sources with harmonic components in the micro grid, by locating more accurately the nodes where such disturbances occur thanks to the geolocation of advanced meters.

J. What are the types of data storage for advanced metering systems?

Advanced metering systems can or cannot have data storage capability, as listed below:

-No storage capacity.

-Local storage inside a metering device.

-Central storage in an external storage device used as processing central.

-Local and central storage.

In some isolated cases, data storage is not required. However, for microgrids, it is necessary either data storage to be sent to the central later or the use of this data to plan possible scenarios and energy flow management in the installation. Additionally, the possibility of storing the data increases the analysis capability, management and central response control towards unexpected communication failures, thus eliminating data loss.

\section{K. $\quad$ What are Distributed Metering Systems?}

A distributed metering system (DMS) within a building is formed by several meters located in the topology of the electrical network. Usually, they are located in the connection point to the network operator, in the main electrical panel, in local electrical panels and/or loads. The DMS helps workers and network supervisors to manage the energy to improve its efficiency, to optimize the network maintenance, to improve predictions and reduce failures, to detect disturbances and problematic zones with THD. For this, it is required that the
DMS can record can process the data obtained with the advanced meters and segregate them by areas and specific services according to the type of building

\section{CONCLUSION}

Microgrids have allowed the integration of different generation technologies and a more active participation of consumers. Currently, they can be seen as the building blocks of the future power grid. Communications and advanced meters are key aspects for such integration for obtaining the best in terms of efficiency and power quality. In regards to communications, recently, wireless technologies have been dominated over wired technologies. LPWAN is more than suitable for communication in microgrids which does not require huge amounts of data transmission or a long range of operation. three LPWAN technologies (Sigfox, LoRa and NBIoT.) were compared in terms of battery duration, latency, messages length, terminal devices quantity, coverage, quality of service, implementation complexity and system cost. Microgrids designers can choose the most convenient LPWAN technology according to their specific requirements.

The advantages of implementing advanced metering and some important concepts regarding this issue were highlighted. In general terms, the possibility of the appropriate integration of DERs nodes into the electric grid, using advanced meters to provide the needed information of local energy consumption, bidirectional energy flows and energy quality variables will help the network operator to make better decisions, and even step ahead a possible system failure. Additionally, the incorporation of distributed metering systems would provide tools for improving and managing power networks and microgrids, it will be possible to improve its efficiency, to optimize the network maintenance, to improve predictions and reduce failures, to detect disturbances and to increase power quality.

\section{ACKNOWLEDGEMENTS}

The authors gratefully acknowledge the support from the Colombia Scientific Program within the framework of the call Ecosistema Científico (Contract No. FP44842- 218-2018). The authors also want to acknowledge Universidad de Antioquia for its support through the project "Estrategia de Sostenibilidad"

\section{REFERENCES}

[1] R. A. Kaushik y N. M. Pindoriya, «Power flow control of hybrid AC-DC microgrid using master-slave technique», en 2014 IEEE Conference on Energy Conversion (CENCON), 2014, pp. 389-394, doi: 10.1109/CENCON.2014.6967535.

[2] X. Shi, J. Zhu, L. Li, y Y. Qu, «Model predictive control of PWM AC/DC converters for Bi-directional power flow control in microgrids», en 2015 Australasian Universities Power Engineering Conference (AUPEC), 2015, pp. 1-4, doi: 


\subsection{9/AUPEC.2015.7324892.}

[3] H. Lotfi y A. Khodaei, «AC Versus DC Microgrid Planning», IEEE Trans. Smart Grid, vol. 8, n. ${ }^{\circ}$ 1, pp. 296-304, ene. 2017, doi: 10.1109/TSG.2015.2457910.

[4] Z. Guoping, W. Weijun, y M. Longbo, «An Overview of Microgrid Planning and Design Method», en 2018 IEEE 3rd Advanced Information Technology, Electronic and Automation Control Conference (IAEAC), 2018, pp. 326-329, doi: 10.1109/IAEAC.2018.8577763.

[5] S. Ruiz, J. Patiño, A. Marquez-Ruiz, J. Espinosa, E. Duque, y P. Ortiz, «Optimal Design of a Diesel-PVWind-Battery-Hydro Pumped POWER system with the Integration of ELECTRIC vehicles in a Colombian Community», Energies, vol. 12, n. ${ }^{\circ} 23$, p. 4542, ene. 2019, doi: 10.3390/en12234542.

[6] M. Khalid, «A Review on the Selected Applications of Battery-Supercapacitor Hybrid Energy Storage Systems for Microgrids», Energies, vol. 12, n. ${ }^{\circ}$ 23, p. 4559, ene. 2019, doi: 10.3390/en12234559.

[7] A. Castellazzi, E. Gurpinar, Z. Wang, A. Suliman Hussein, y P. Garcia Fernandez, "Impact of WideBandgap Technology on Renewable Energy and SmartGrid Power Conversion Applications Including Storage», Energies, vol. 12, n. ${ }^{\circ} 23$, p. 4462, ene. 2019, doi: 10.3390/en12234462.

[8] W. Yu, S. Li, Y. Zhu, y C.-F. Yang, «Management and Distribution Strategies for Dynamic Power in a Ship's Micro-Grid System Based on Photovoltaic Cell, Diesel Generator, and Lithium Battery», Energies, vol. 12, n. ${ }^{\circ}$ 23, p. 4505, ene. 2019, doi: 10.3390/en12234505.

[9] L. G. Marín et al., «Hierarchical Energy Management System for Microgrid Operation Based on Robust Model Predictive Control», Energies, vol. 12, n. ${ }^{\circ} 23$, p. 4453, ene. 2019, doi: 10.3390/en12234453.

[10] G.-K. Chang, W. Jian, Z. Jia, y A. Chowdhury, «Broadband access technologies for very high throughput wireless sensor communication networks», en 2010 IEEE Radio and Wireless Symposium (RWS), 2010, pp. 496-499, doi: 10.1109/RWS.2010.5434110.

[11] M. Islam y H.-H. Lee, «Microgrid communication network with combined technology», en 2016 5th International Conference on Informatics, Electronics and Vision (ICIEV), 2016, pp. 423-427, doi: 10.1109/ICIEV.2016.7760039.

[12] S. Rauh, J. Robert, M. Schadhauser, y A. Heuberger, «LPWAN occupancy model parameter identification for license exempt sub-GHz frequency bands», en 2018 11th German Microwave Conference (GeMiC), 2018, pp. 111-114, doi: 10.23919/GEMIC.2018.8335041.

[13] A. Kosari y D. D. Wentzloff, «MURS Band for LPWAN Applications», en 2019 IEEE Topical Conference on Wireless Sensors and Sensor Networks (WiSNet), 2019, pp. 1-3, doi: 10.1109/WISNET.2019.8711814.

[14] K. Mekki, E. Bajic, F. Chaxel, y F. Meyer, «A comparative study of LPWAN technologies for largescale IoT deployment», ICT Express, vol. 5, n. ${ }^{\circ}$ 1, pp. 1-7, mar. 2019, doi: 10.1016/j.icte.2017.12.005.

[15] M. Centenaro, L. Vangelista, A. Zanella, y M. Zorzi, «Long-range communications in unlicensed bands: the rising stars in the IoT and smart city scenarios», IEEE Wirel. Commun., vol. 23, n. ${ }^{\circ}$ 5, pp. 60-67, oct. 2016, doi: 10.1109/MWC.2016.7721743.

[16] U. Raza, P. Kulkarni, y M. Sooriyabandara, «Low Power Wide Area Networks: An Overview», IEEE Commun. Surv. Tutor., vol. 19, n. ${ }^{\circ}$ 2, pp. 855-873, Secondquarter 2017, doi: 10.1109/COMST.2017.2652320.

[17] A. M. Baharudin y W. Yan, «Long-range wireless sensor networks for geo-location tracking: Design and evaluation», en 2016 International Electronics Symposium (IES), 2016, pp. 76-80, doi: 10.1109/ELECSYM.2016.7860979.

[18] W. Guibene, J. Nowack, N. Chalikias, K. Fitzgibbon, M. Kelly, y D. Prendergast, «Evaluation of LPWAN Technologies for Smart Cities: River Monitoring UseCase», en 2017 IEEE Wireless Communications and Networking Conference Workshops (WCNCW), 2017, pp. 1-5, doi: 10.1109/WCNCW.2017.7919089.

[19] O. Vondrouš, Z. Kocur, T. Hégr, y O. Slavíček, «Performance evaluation of IoT mesh networking technology in ISM frequency band», en 2016 17th International Conference on Mechatronics Mechatronika (ME), 2016, pp. 1-8.

[20] M. Moghimi, C. Bennett, D. Leskarac, S. Stegen, y J. $\mathrm{Lu}$, «Communication architecture and data acquisition for experimental MicroGrid installations», en 2015 IEEE PES Asia-Pacific Power and Energy Engineering Conference (APPEEC), 2015, pp. 1-5, doi: 10.1109/APPEEC.2015.7380917.

[21] Y. Ben-Shimol, S. Greenberg, y K. Danilchenko, «Application-Layer Approach for Efficient Smart Meter Reading in Low-Voltage PLC Networks», IEEE Trans. Commun., vol. 66, n. ${ }^{\circ}$ 9, pp. 4249-4258, sep. 2018, doi: 10.1109/TCOMM.2018.2828849.

[22] B. Botte, V. Cannatelli, y S. Rogai, «The Telegestore project in Enel's metering system», en CIRED 2005 18th International Conference and Exhibition on Electricity Distribution, 2005, pp. 1-4, doi: 10.1049/cp:20051383.

[23] J. Duplex, S. Gosswiller, y S. Fagnoni, «A better knowledge of electricity consumption for residential customers through the Linky smart meter», en 22nd International Conference and Exhibition on Electricity Distribution (CIRED 2013), 2013, pp. 1-4, doi: 10.1049/cp.2013.1188.

[24] N. Andreadou, M. O. Guardiola, y G. Fulli, «Telecommunication Technologies for Smart Grid Projects with Focus on Smart Metering Applications», 2016, doi: 10.3390/en9050375.

[25] Xiaolin Lu, Yanjun Sun, y Il Han Kim, «Reliable power line communication - A vehicle to smart home 
International Journal of Engineering Research and Technology. ISSN 0974-3154, Volume 13, Number 9 (2020), pp. 2196-2204

(C) International Research Publication House. https://dx.doi.org/10.37624/IJERT/13.9.2020.2196-2204

and smart energy», en 2012 IEEE International Conference on Consumer Electronics (ICCE), 2012, pp. 86-87, doi: 10.1109/ICCE.2012.6161752.

[26] S. Oh, J. S. Haberl, y J.-C. Baltazar, «Analysis methods for characterizing energy saving opportunities from home automation devices using smart meter data», Energy Build., p. 109955, mar. 2020, doi: 10.1016/j.enbuild.2020.109955.

[27] E. Rodriguez-Diaz, E. J. Palacios-García, M. Savaghebi, J. C. Vasquez, J. M. Guerrero, y A. Moreno-Munoz, «Advanced smart metering infrastructure for future smart homes», en 2015 IEEE 5th International Conference on Consumer Electronics - Berlin (ICCE-Berlin), 2015, pp. 29-31, doi: 10.1109/ICCE-Berlin.2015.7391260.

[28] H.-J. Choi y J.-H. Jung, «Enhanced Power Line Communication Strategy for DC Microgrids Using Switching Frequency Modulation of Power Converters», IEEE Trans. Power Electron., vol. 32, n. ${ }^{\circ}$ 6, pp. 4140-4144, jun. 2017, doi: 10.1109/TPEL.2017.2648848.

[29] T. K. TRAN, D. GENON-CATALOT, y H. YAHOUI, «DC Microgrid implementation based on Powerline Communication (PLC)», en 2019 IEEE 4th International Future Energy Electronics Conference (IFEEC), 2019, pp. 1-5, doi: 10.1109/IFEEC47410.2019.9015034.

[30] N. Uribe-Pérez et al., «Smart management of a distributed generation microgrid through PLC PRIME technology», en 2015 International Symposium on Smart Electric Distribution Systems and Technologies (EDST), 2015, pp. 374-379, doi: 10.1109/SEDST.2015.7315237.

[31] D. Sharma, R. K. Mallik, S. Mishra, A. Dubey, y V. Ranjan, "Voltage control of a DC microgrid with double-input converter in a multi-PV scenario using PLC», en 2016 IEEE Power and Energy Society General Meeting (PESGM), 2016, pp. 1-5, doi: 10.1109/PESGM.2016.7741500.

[32] Y. Chung, J. Y. Ahn, y J. Du Huh, «Experiments of A LPWAN Tracking(TR) Platform Based on Sigfox Test Network», en 2018 International Conference on Information and Communication Technology Convergence (ICTC), 2018, pp. 1373-1376, doi: 10.1109/ICTC.2018.8539697.

[33] A. Lavric y V. Popa, «Internet of Things and LoRa ${ }^{\mathrm{TM}}$ Low-Power Wide-Area Networks: A survey», en 2017 International Symposium on Signals, Circuits and Systems (ISSCS), 2017, pp. 1-5, doi: 10.1109/ISSCS.2017.8034915.

[34] D. Zorbas, K. Abdelfadeel, P. Kotzanikolaou, y D. Pesch, «TS-LoRa: Time-slotted LoRaWAN for the Industrial Internet of Things», Comput. Commun., vol. 153, pp. 1-10, mar. 2020, doi: 10.1016/j.comcom.2020.01.056.

[35] L. Li, J. Ren, y Q. Zhu, «On the application of LoRa LPWAN technology in Sailing Monitoring System», en
2017 13th Annual Conference on Wireless On-demand Network Systems and Services (WONS), 2017, pp. 77 80, doi: 10.1109/WONS.2017.7888762.

[36] A. Zourmand, A. L. Kun Hing, C. Wai Hung, y M. AbdulRehman, «Internet of Things (IoT) using LoRa technology», en 2019 IEEE International Conference on Automatic Control and Intelligent Systems (I2CACIS), 2019, pp. 324-330, doi: 10.1109/I2CACIS.2019.8825008.

[37] Y. Lu, Y. Liu, C. Hu, J. Xu, Z. Wang, y S. Chen, «LoRa-based Communication Technology for Overhead Line Internet of Things», en 2019 4th International Conference on Intelligent Green Building and Smart Grid (IGBSG), 2019, pp. 471-474, doi: 10.1109/IGBSG.2019.8886176.

[38] R. Ratasuk, B. Vejlgaard, N. Mangalvedhe, y A. Ghosh, «NB-IoT system for M2M communication», en 2016 IEEE Wireless Communications and Networking Conference Workshops (WCNCW), 2016, pp. 428-432, doi: 10.1109/WCNCW.2016.7552737.

[39] S. Foni, T. Pecorella, R. Fantacci, C. Carlini, P. Obino, y M.-G. Di Benedetto, «Evaluation methodologies for the NB-IOT system: Issues and ongoing efforts», en 2017 AEIT International Annual Conference, 2017, pp. 1-6, doi: 10.23919/AEIT.2017.8240557.

[40] A. Ikpehai et al., «Low-Power Wide Area Network Technologies for Internet-of-Things: A Comparative Review», IEEE Internet Things J., vol. 6, n. ${ }^{\circ}$ 2, pp. 2225-2240, abr. 2019, doi: 10.1109/JIOT.2018.2883728.

[41] F. C. de Oliveira, J. J. P. C. Rodrigues, R. A. L. Rabêlo, y S. Mumtaz, «Performance Delay Comparison in Random Access Procedure for NB-IoT, LoRa, and SigFox IoT Protocols», en 2019 IEEE 1st Sustainable Cities Latin America Conference (SCLA), 2019, pp. 16, doi: 10.1109/SCLA.2019.8905443.

[42] G. R. Barai, S. Krishnan, y B. Venkatesh, «Smart metering and functionalities of smart meters in smart grid - a review», en 2015 IEEE Electrical Power and Energy Conference (EPEC), 2015, pp. 138-145, doi: 10.1109/EPEC.2015.7379940.

[43] V. G. Vilas, A. Pujara, S. M. Bakre, y V. Muralidhara, «Implementation of metering practices in smart grid», en 2015 International Conference on Smart Technologies and Management for Computing, Communication, Controls, Energy and Materials (ICSTM), 2015, pp. 484-487, doi: 10.1109/ICSTM.2015.7225465.

[44] M. Carratù, M. Ferro, A. Pietrosanto, y V. Paciello, «Smart Power Meter for the IoT», en 2018 IEEE 16th International Conference on Industrial Informatics (INDIN), 2018, pp. 514-519, doi: 10.1109/INDIN.2018.8472018.

[45] N. S. Živic, O. Ur-Rehman, y C. Ruland, «Evolution of smart metering systems», en 2015 23rd Telecommunications Forum Telfor, 2015, pp. 635-638, doi: 10.1109/R.2015.7377547. 
International Journal of Engineering Research and Technology. ISSN 0974-3154, Volume 13, Number 9 (2020), pp. 2196-2204 (C) International Research Publication House. https://dx.doi.org/10.37624/IJERT/13.9.2020.2196-2204

[46] P. Bansal y A. Singh, «Smart metering in smart grid framework: A review», en 2016 Fourth International Conference on Parallel, Distributed and Grid Computing (PDGC), 2016, pp. 174-176, doi: 10.1109/PDGC.2016.7913139. 